\title{
Ideas, preguntas y explicaciones de los niños sobre el cielo de Bogotá*
}

IDEAS, QUESTIONS AND EXPLANATIONS OF CHILDREN ON SKY BOGOTÁ

च IDÉIAS, PERGUNTAS E EXPLICAÇÕES DE CRIANÇAS NO CÉU BOGOTÁ

\section{Carlos Andrés Castañeda Sua ${ }^{\star *}$ / and3cas@gmail.com}

\section{Resumen}

En el presente artículo se expone una inserción en el aula, realizada por el maestro, para abordar las ciencias naturales mediante el estudio de la Astronomía; se presentan actividades efectuadas en torno a las ideas, preguntas y explicaciones de los estudiantes sobre algunos eventos y situaciones del entorno físico y natural. Por último, se distinguen estrategias para vincular las vivencias del estudiante.

\section{Summary}

This article shows an insertion in the classroom by the teacher, for the approach of the natural sciences through the study of Astronomy activities around ideas, questions and explanations of students on some events occur and situations of physical and natural environment. Distinctive strategies to link the experiences of the student.
Palabras clave

Ideas, preguntas, explicaciones, Astronomía, enseñanza.

\section{Resumo}

Este artigo mostra uma inserção na sala de aula pelo professor, para a abordagem das ciências naturais, através do estudo das atividades de Astronomia em torno de idéias, perguntas e explicações dos alunos sobre alguns eventos ocorrem e situações de ambiente físico e natural. Estratégias distintas de relacionar as experiências do aluno.

\section{Keywords}

Ideas, questions, explanations, Astronomy, teaching.

Palavras chave

Ideias, perguntas, explicações, Astronima, ensino.

\footnotetext{
* El artículo hace parte de la monografía "Mirada al cielo de Bogotá: Una experiencia con niños de quinto grado de primaria", orientada por Rosa I. Pedreros M., profesora del Departamento de Física de la Universidad Pedagógica Nacional, 2012.

** Licenciado en Física, Universidad Pedagógica Nacional.
}

Fecha de recepción: 29 de marzo de 2014 / Fecha de aprobación: mayo 23 de 2014 


\section{Introducción}

Cuando consideramos la necesidad de constituir espacios en el aula en los cuales podamos distinguir el mundo de posibilidades de la enseñanza de la Astronomía, pensamos inicialmente en la construcción de escenarios con características que permitan relacionar los diferentes elementos que se encuentran en la base de la enseñanza y el aprendizaje de este ámbito del conocimiento, que fortalezcan al estudiante en su deseo de saber y aprender, y enriquezcan la mirada pedagógico-didáctica, disciplinar y epistemológica del profesor al pensar las propuestas de clase.

Constituir estos espacios significa partir de la idea de que la Astronomía es una ciencia que apasiona a muchas personas y genera interés por los sucesos que ocurren en el cielo; muchos de estos eventos suscitan preguntas y, con ellas, la búsqueda de explicaciones. Los espacios que favorecen el estudio de la Astronomía posibilitan el aumento de la curiosidad, el deseo de saber y una actitud investigativa de estudiantes y maestros, fomentando su participación en clase; a la vez que enriquecen la mirada acerca de la ciencia y el valor de su enseñanza.

\section{Investigaciones sobre la Astronomía y su enseñanza}

Existe una amplia gama de profesores e investigadores interesados y apasionados por la explicación e investigación de la Astronomía, su enseñanza y el esclarecimiento de los fenómenos que atañen a este campo. Como lo expone Camino (1999), una de las características más destacables de estos estudios es que se pueden realizar observaciones astronómicas sin instrumentos costosos o complicados de manejar, lo cual no significa que no se deban enfrentar dificultades a la hora de enseñar la materia.

Es por ello que Ros (2009) considera importante reconocer algunas de las posibles dificultades a la hora de trabajar la Astronomía en el aula; por ejemplo, el hecho de que un gran número de profesores no tiene el bagaje suficiente para tratar los asuntos astronómicos y, mu- chas veces, esto lleva a pensar que si no se cuenta con un buen telescopio no se puede llevar a cabo ninguna actividad. Para desvirtuar esta creencia, Ros (2009) presenta algunas recomendaciones para conseguir buenos resultados en cuanto al aprovechamiento de la didáctica de la observación, entre ellas, la posibilidad de abordar temas que despierten la creatividad y el interés de los estudiantes, pero que a su vez tengan una relación con otras áreas del conocimiento, como las matemáticas.

Es desde esta perspectiva que Pérez (2009) realiza un análisis de la historia de la Astronomía, involucrando las nuevas tecnologías de la información y la comunicación (TIC's) para, desde allí, comprender y confirmar algunas de las teorías realizadas en siglos anteriores. En el mismo sentido, Arias (2006), Sánchez (1997) y Barbosa y Ramírez (1995), presentan posibles estrategias de aula que permiten la comprensión de algunos conceptos como el movimiento, tamaño, distancia y posición, mediante la observación y la discusión en el aula.

En el ámbito latinoamericano, la Astronomía es una materia obligatoria en las escuelas y colegios de Uruguay desde 1989, y se ofrece desde la posibilidad de vincular temas de la ciencia con las clases de básica primaria, debido a que permite la relación con temas desarrollados en las distintas asignaturas presentes en el plan de estudio. Otro pionero en el manejo de la materia es Argentina; allí se plantean estrategias para vincular la Astronomía a la escuela primaria, desde propuestas que presentan el modelo geocéntrico como una herramienta eficaz para la enseñanza de los movimientos astrales, pues permite generar en los estudiantes de primaria una relación con las observaciones directas de los fenómenos celestes; sin embargo, es necesario considerar que muchos de los eventos se analizan desde una mirada externa a la Tierra, y los estudiantes no la logran familiarizarse completamente, se confunden u olvidan con facilidad las explicaciones que se presentan.

Las experiencias mencionadas permiten identificar una búsqueda de estrategias y metodologías para pensar la Astronomía y su enseñanza, en ellas se tienen en cuenta las inquietudes que emergen de la cotidianidad y la interacción de los estudiantes con el mundo físico y natural. Así, la enseñanza de la Astronomía, particu- 
larmente en la Educación Básica Primaria, se constituye como opción para abordar el trabajo en torno a las ciencias naturales, pero desde la perspectiva de hacer de la ciencia algo entretenido e interesante, y de pensar la clase como un espacio para la comprensión de lo que se estudia; así se propiciarán diversas vivencias de conocimiento en el aula. Esto es posible si se tienen en cuenta las ideas, preguntas y situaciones cotidianas que sean del interés de los estudiantes. Si se atiende el deseo de saber y de conocer, será posible desplegar un aprendizaje significativo en la clase de ciencias, especialmente para la Astronomía.

\section{Referentes}

El trabajo que se socializa en el presente artículo contempla cuatro referentes: la idea o imagen de ciencia; el trabajo alrededor de las ideas de los niños; el trabajo alrededor de las preguntas de los niños; y la elaboración de explicaciones. Cada de uno de estos aspectos se expone a continuación.

Idea o imagen de ciencia: la ciencia se desarrolla a partir de los contextos social, político y económico, que influyen en la generación de nuevas teorías; la historia de las ciencias da cuenta de este fenómeno, pues la presentación de las tesis que buscaban explicar algunos eventos astronómicos, como la teoría heliocentrista y geocentrista, tuvo distintos impactos que variaron de acuerdo con el arraigo de determinadas creencias en la comunidad; estas creencias influyen al momento de que una teoría sea más o menos aceptada, como lo afirma Ayala (2005, p. 9):

"La ciencia es concebida como una actividad de comprensión del mundo que, de acuerdo a contextos socio-culturales específicos y dando respuesta a éstos, desarrolla una comunidad que se ha venido constituyendo históricamente, legitimándose socialmente, generando tradiciones y recomponiéndose de acuerdo a las dinámicas de las condiciones históricas".

La ciencia depende de factores socio-culturales para su aceptación y asimilación; al tratar de modificar o cambiar el modo de concebir o explicar un evento del entorno natural, se tiende a presentar una negación entre los individuos que conforman el entorno social.
El trabajo en torno a las ideas de los niños: las ideas de los estudiantes parten de su experiencia con los hechos y eventos físicos y naturales, como lo afirma Cubero (1989 p. 12):

"[...] los estudiantes adquieren ideas de cómo son los hechos y fenómenos sociales y naturales mediante su experiencia con todo lo que les rodea, lo que escuchan y discuten con otras personas o lo que conocen por los medios de comunicación; muchos de estos hechos serán objeto de estudio a lo largo de la escolarización".

Las ideas de los niños no se dan al azar, están relacionadas con lo que ya conocen, con su pensamiento y su experiencia en un ambiente socio-cultural. Sus ideas tienen total validez para ellos, y es necesario que el profesor las conozca para que las vincule a las explicaciones e ideas que se presentan en la historia de las ciencias; no es aconsejable aislarlas, pues se impide que la comprensión del estudiante tenga mejores resultados académicos.

En este sentido, es necesario comprender que los estudiantes no son una hoja en blanco que se va a llenar con la clase, porque desde sus primeros años tienen experiencias y éstas les permiten describir los sucesos y eventos que ocurren en su entorno; si lo que se desea es lograr un aprendizaje significativo, no se deben pasar por alto las ideas de los estudiantes, por el contrario, los docentes deben apropiarse de ellas para mejorar las prácticas pedagógicas que permiten la comprensión, el interés y el desarrollo de la creatividad de los estudiantes (Cubero, 1989).

El trabajo en torno a las preguntas: las preguntas juegan un papel importante al momento de generar conocimiento, no solo en el aula de clase, sino en cualquier ámbito de la vida en general; sin embargo, en la sociedad se ha dejado de lado la curiosidad y la generación de preguntas que permitan profundizar, como lo afirman Giordan y Vecchi (1995): "Toda nuestra cultura nos impide plantearnos preguntas, pues éstas ya tienen respuestas. Por ejemplo, los periodistas son curiosos por nosotros, se preguntan por nosotros, debaten por nosotros..., piensan por nosotros" (p. 172). 
La curiosidad está estrechamente relacionada con el deseo de conocer y aprender, pero la sociedad y la academia han abandonado este principio fundamental; esto no significa que las preguntas no estén presentes en la escuela, sino que su proposición está a cargo del profesor, quien las utiliza para generar interés en sus estudiantes. El problema radica en que en ocasiones sus preguntas no responden a las motivaciones ni a la actividad académica, y se convierten en un ejercicio que busca adivinar la repuesta que desea el docente, antes que en una propuesta real para comprender la situación problema.

Esto sucede debido a que los interrogantes propuestos no motivan, ni interesan a la clase, Giordn y Vechhi se refieren a la situación en los siguientes términos: "no se debe obligar a aprender a un alumno aquello por lo cual no se siente curiosidad. Es mediante este sentimiento como el alumno intenta buscar una información que responde a su necesidad real de explicación" (1995, p. 178); por tal razón, es necesario tener en cuenta las preguntas que surgen del interés de los estudiantes en la clase, ya que posibilitan una mayor apropiación de los espacios académicos. Las inquietudes de los estudiantes pueden ser muy dispersas y variadas, lo cual no supone su no viabilidad; por el contrario, es aquí donde el profesor debe intervenir encontrando preguntas comunes que permitan un interés mayor por parte de la clase. Las preguntas deben responder a los intereses colectivos.

Elaboración de explicaciones: las reflexiones de las experiencias pedagógico-didácticas, nacidas desde la perspectiva de la enseñanza de la Astronomía, permiten identificar énfasis, avances y estudios particulares teniendo en cuenta eventos del mundo físico, en particular del cielo de Bogotá. Para verlas en plenitud es necesario reconocer las explicaciones de profesores y estudiantes, ya que son el eje fundamental de la ciencia, pues permite entender los eventos que, desde su perspectiva, se presentan en el mundo natural.

Al respecto, Segura (1993, p. 5), afirma que: "lo que realmente diferenció a los griegos de babilónicos y egipcios no fue la habilidad para realizar nuevas observaciones, sino su preocupación por inventarse nuevas cosmovisiones que permitan explicar las ob- servaciones y racionalizar las predicciones". Así, tener una gran cantidad de información no garantiza generar conocimiento, la explicación que se genere a partir de esta información es lo que realmente permite hacerlo.

En un primer momento, las explicaciones surgen de forma individual, teniendo en cuenta los sistemas ideológicos presentes en el estudiante; para que emerjan, es necesario generar cambios en la forma en que se piensa el mundo, esto permite observar las diferentes formas usadas por cada individuo para interpretar el mismo evento, y su validez dependerá de la postura asume respecto del mismo.

Desde esta perspectiva, un factor importante en la presente investigación es, como lo afirman Bautista y Rodríguez (1996, p. 67): "Formar personas que puedan desarrollar la actividad de producción de conocimiento más que la simple adquisición o distribución de los mismos". Lo que se busca es que los estudiantes sean críticos con la información que se les presente, que puedan hacer relaciones entre lo que se presenta y el mundo natural que los rodea; en general, que se produzca un conocimiento colectivo en el que el punto en común sea el interés por comprender, antes que una imposición del maestro.

\section{Metodología}

En cuanto a los aspectos metodológicos, se tuvo en cuenta la perspectiva cualitativa con enfoque interpretativo (Rodríguez, Gómez, Flores y Jiménez, 1996). Se toma la perspectiva interpretativa porque trata de aproximarse al universo interpretativo del otro, a la cultura del "otro" como sujeto enmarcado en unas condiciones históricas, sociales y culturales específicas (Molina, 2000).

Las técnicas utilizadas para la recolección de información fueron el grupo focal, que propiciaba el intercambio de ideas; para ello se tuvo en cuenta que las personas que hicieron parte del mismo tuvieran aspectos comunes (niños de quinto de primaria). También se usaron las narrativas, que emergen de los testimonios organizados de los estudiantes; éstas constituyen las historias personales y las soluciones a situaciones, 


\section{IIIII}

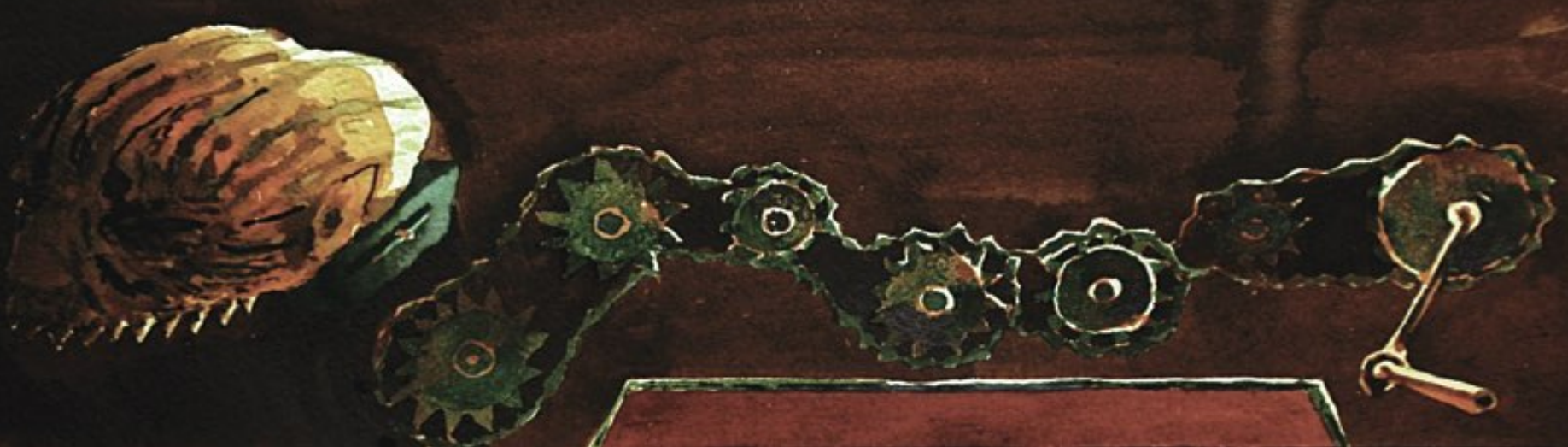

Luis Antonio Sánchez León » Título: Nadie Ve Nadie Escucha » Técnica: Acuarela Y Tinta » Tamaño: 35 X 16.2 Cm 》 Año: 2014 
también resultan pertinentes porque permiten evidenciar y organizar la experiencia de los sujetos, sus conocimientos, las negociaciones con el mundo social y natural, los valores, los criterios estéticos y otros aspectos valorativos. Los instrumentos empleados fueron las hojas de los estudiantes y de trabajo en grupo, frisos construidos y la bitácora del maestro.

La experiencia se realizó con los niños de quinto de primaria del Centro Integral Distrital José María Córdoba I.E.D, jornada mañana, ubicado en la localidad de Tunjuelito. El grupo estaba conformado por treinta y cinco (35) estudiantes, quince (15) niñas y veinte (20) niños, sus edades oscilaban entre los 9 y 12 años. Las categorías utilizadas en el análisis de las actividades fueron:

Ideas: con esta categoría se pretenden encontrar las formas con las que se entienden los eventos del mundo natural, aquellos relacionados con lo que suscita en el cielo.

Preguntas: en esta categoría se destacan los temas que generan interés de los estudiantes por la Astronomía; se trabajaron mediante preguntas que suscitan la explicación.

Explicaciones: mediante esta categoría se observa la forma en que los estudiantes abordan una situación o evento e intentan darle respuesta mediante lo que comprenden; también se ven las comparaciones que hacen de otros contextos.

\section{Descripción y resultados de la actividad en el aula}

Desde los inicios de la humanidad se ha contemplado el cielo, personas y comunidades de todas las épocas se han detenido un instante a hacerlo. En la vida cotidiana los niños, jóvenes y adultos se han preguntado o han escuchado: ¿por qué nos sigue la luna?; ¿por qué se presentan las fases lunares?; ¿por qué a veces podemos ver la luna y el sol de día?; ¿por qué el día y la noche?; ¿cómo se originó el universo?; ¿hay vida en otros planetas o en otro lugar del espacio?; ¿cómo se orientaban los antiguos?; ¿cómo se diseñaron los calendarios?
Estas y muchas otras preguntas suscitan interés e inquietudes el entorno. Astrónomos, pensadores y científicos han estudiado estos eventos y procesos naturales a través de la historia de la ciencia. En particular, en el presente trabajo se parte de las ideas, preguntas y situaciones que desean abordar los estudiantes, y la actividad tuvo como intenciones:

Cultivar la curiosidad, el deseo por saber y construir explicaciones a eventos que nos susciten interés en el ámbito de la Astronomía.

Cuestionarnos sobre el sentido de la Astronomía en la institución escolar y en la vida cotidiana, dado que nuestra idea de Astronomía genera unas formas de proceder en la clase, una manera de plantear y de abordar las actividades en el aula.

Involucrarnos colectivamente en la búsqueda de alternativas que tengan sentido para cada uno de los estudiantes, de tal manera que se generen procesos investigativos en el ámbito de la Astronomía, en particular en las escuelas de nuestros contextos locales, y se propicien vivencias de conocimiento con los estudiantes a partir de la elaboración de explicaciones de los eventos abordados en clase.

\section{Alternativa metodológica en el aula}

Se tuvo en cuenta la alternativa metodológica Actividades Totalidad Abiertas -ATAs- (Segura, Molina, Pedreros, Arcos, Velasco, Leuro y Hernández, 1995), pues permite cumplir con la coherencia conceptual, es decir, la manera como se articulan los conocimientos que se construyen con los conocimientos anteriores que poseen los estudiantes (problema epistemológico); también posibilita la coherencia lógica, la selección de los temas que se tratan y la determinación de su profundidad, en cuanto que ésta debe corresponder, entre otras, al desarrollo intelectual del estudiante (posibilidades de comprensión-elaboración de discurso, problema lógico); por último, facilita el desarrollo de la coherencia en el formato de la actividad, es decir, la selección de los temas o problemas que se resuelven en la clase, en cuanto a la actitud de los estudiantes frente a ellos (por ejemplo, relaciones de apropiaciónrechazo), pues son determinantes para la captura del interés por lo que se hace (problema de pertinencia).

96 nodos y nudos / volumen 4 N.o 36 / enero - junio / 2014 ISSN: 0122-4328 / p.p 91-104 
En el desarrollo de la propuesta de aula se tuvieron en cuenta estrategias como: el trabajo alrededor de las ideas e inquietudes; la organización de la actividad a partir de las preguntas; la investigación del estudiante; el estudio de situaciones, y la elaboración de explicaciones, comunicación y divulgación de la vivencia en el aula. La dinámica en el aula contempla el trabajo en grupo, momentos colectivos para llevar a cabo el proceso de elaboración de explicaciones por parte de los estudiantes, y diversos talleres para enriquecer las búsquedas individuales y colectivas. Las sesiones de trabajo se recogen en la Tabla 1.

Tabla 1. Sesiones de trabajo en el aula

\begin{tabular}{|c|c|c|c|}
\hline Sesión & Nombre & Descripción e intención & Actividad \\
\hline \multirow{2}{*}{$\stackrel{\frac{\pi}{2}}{\stackrel{5}{\frac{5}{2}}}$} & $\begin{array}{l}\text { Reconocimiento de la } \\
\text { clase y expectativas } \\
\text { de las actividades }\end{array}$ & $\begin{array}{l}\text { Se busca interactuar con los niños para } \\
\text { empezar a conocerlos y distinguirlos }\end{array}$ & $\begin{array}{l}\text { Se realizará un conversatorio } \\
\text { entre los estudiantes } \\
\text { y el maestro }\end{array}$ \\
\hline & Exploración de ideas & $\begin{array}{l}\text { Se propicia un diálogo con los estudiantes } \\
\text { para explorar las ideas, lo que quieren } \\
\text { saber, lo que les interesa estudiar, etc. }\end{array}$ & $\begin{array}{l}\text { Se recogen las ideas en hojas } \\
\text { de trabajo de los niños y en } \\
\text { la bitácora del maestro }\end{array}$ \\
\hline 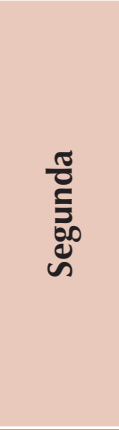 & $\begin{array}{l}\text { Organización de las } \\
\text { inquietudes y preguntas } \\
\text { de los estudiantes }\end{array}$ & $\begin{array}{l}\text { Identificar los eventos de mayor interés de } \\
\text { los estudiantes y generar grupos de trabajo } \\
\text { para abordar las temáticas de interés } \\
\text { Conocer las ideas y conceptos } \\
\text { que tienen acerca del tema a tratar } \\
\text { como referente de partida } \\
\text { Enriquecer la mirada sobre los asuntos } \\
\text { que desean conocer los estudiantes }\end{array}$ & $\begin{array}{l}\text { Presentación y distinción } \\
\text { de las preguntas e } \\
\text { inquietudes, agrupándolas } \\
\text { según temas de interés }\end{array}$ \\
\hline$\frac{\pi}{\frac{\pi}{2}}$ & $\begin{array}{c}\text { Trabajo en torno a } \\
\text { inquietudes y preguntas }\end{array}$ & $\begin{array}{l}\text { Trabajar en torno a las inquietudes y preguntas } \\
\text { se constituye en un ámbito del conocimiento; } \\
\text { posibilita organizar las actividades en el } \\
\text { aula en el ámbito de la Astronomía, de tal } \\
\text { manera que responda a los intereses de los } \\
\text { estudiantes y del maestro a cerca de los } \\
\text { eventos y sucesos de nuestro entorno, en } \\
\text { particular, los relacionados con nuestro cielo } \\
\text { de Bogotá. Por ejemplo: ¿Por qué las estrellas } \\
\text { no se mueven?; ¿cómo se crearon las estrellas? }\end{array}$ & $\begin{array}{l}\text { Inicio de la exploración } \\
\text { colectiva de acuerdo a los } \\
\text { intereses o inquietudes } \\
\text { Elaboración individual y grupal } \\
\text { de las primeras reflexiones } \\
\text { Formulación de preguntas } \\
\text { sobre el objeto de estudio }\end{array}$ \\
\hline$\frac{\pi}{3}$ & $\begin{array}{l}\text { Modos de hablar y } \\
\text { mostrar el asunto de } \\
\text { estudio de la clase }\end{array}$ & $\begin{array}{c}\text { Conocer las dificultades e identificar } \\
\text { situaciones y/o problemas. Este hecho genera } \\
\text { la búsqueda de comprensión, la elaboración } \\
\text { de explicativos y la conceptualización } \\
\text { sobre los eventos astronómicos, cultivando } \\
\text { la curiosidad y el deseo por saber }\end{array}$ & $\begin{array}{l}\text { Exposición de avances acerca } \\
\text { del asunto de estudio } \\
\text { Mostrar los interrogantes a tratar } \\
\text { a los demás grupos de trabajo }\end{array}$ \\
\hline
\end{tabular}




\begin{tabular}{|c|c|c|c|}
\hline Sesión & Nombre & Descripción e intención & Actividad \\
\hline : & $\begin{array}{l}\text { Enriquecimiento de las } \\
\text { búsquedas académicas }\end{array}$ & $\begin{array}{c}\text { Enriquecer la mirada del estudiante y } \\
\text { fortalecer la elaboración de explicaciones. } \\
\text { Se realizarán diversos talleres }\end{array}$ & $\begin{array}{l}\text { Elaboración de talleres } \\
\text { relacionados con eventos } \\
\text { estudiados por los niños } \\
\text { Socialización colectiva de } \\
\text { la vivencia de la sesión }\end{array}$ \\
\hline$\stackrel{\frac{\pi}{x}}{\stackrel{\hbar}{\sharp ~}}$ & $\begin{array}{l}\text { Elaboración de } \\
\text { explicaciones sobre } \\
\text { los asuntos de estudio } \\
\text { de cada equipo de } \\
\text { trabajo en el aula }\end{array}$ & $\begin{array}{l}\text { Realizar exposiciones por parte de } \\
\text { los estudiantes para distinguir las } \\
\text { elaboraciones y comprensiones sobre } \\
\text { los eventos que se observan en el } \\
\text { cielo, generando argumentaciones y } \\
\text { explicaciones para la discusión en clase } \\
\text { Propiciar la actividad investigativa en los } \\
\text { estudiantes y la construcción de discurso }\end{array}$ & $\begin{array}{c}\text { Diseño y construcción } \\
\text { maquetas } \\
\text { Exposición de explicaciones } \\
\text { a las inquietudes generadas } \\
\text { en la investigación }\end{array}$ \\
\hline
\end{tabular}

\section{Análisis de la actividad en el aula}

A continuación se expone lo encontrado a propósito de las ideas de los estudiantes, sus preguntas y explicaciones.

\section{Desde las ideas de los estudiantes}

Las ideas que emergieron de los estudiantes son muy diversas presentaban posibles respuestas a las situaciones de su interés, pero en ellas no se cuenta con una respuesta inmediata, las ideas expresadas eran compartidas con sus compañeros (Ver Tabla 2).

\section{Tabla 2. Ideas surgidas en los estudiantes}

\begin{tabular}{|c|c|}
\hline Grupo & Ideas \\
\hline Planetas & $\begin{array}{l}\text { "Sabemos que Saturno tiene una gran fuerza de magnetismo, por } \\
\text { eso cada meteorito que pasa se atrae al planeta" } \\
\text { "Plutón desapareció porque salió de órbita" } \\
\text { "Hay más planetas de los que conocemos, porque hay más galaxias" }\end{array}$ \\
\hline Estrellas & $\begin{array}{l}\text { "Las estrellas siempre están en el cielo, pero el sol las oculta" } \\
\text { "Las estrellas brillan de diferente color porque de este modo se diferencian más" }\end{array}$ \\
\hline Vida extraterrestre & $\begin{array}{l}\text { "Dicen que los extraterrestres viven en Marte, Júpiter y la luna, pero todavía no se sabe" } \\
\text { "Los extraterrestres son de color verde, con ojos verdes y con antenas" }\end{array}$ \\
\hline Luna & $\begin{array}{l}\text { "La luna es bonita, blanca y única en todo el universo" } \\
\text { "La luna nos ayuda para ver en las noches, nos acompaña y tiene cráteres" }\end{array}$ \\
\hline
\end{tabular}


Cometas,

meteoritos y

asteroides
"Los meteoritos son pedazos de piedras que se mueven por la gravedad que

hay en el espacio, lo cual se mueve de forma diferente a los planetas"

"Los asteroides son algo similar a los meteoritos, pero son muy diferentes a los planetas"

Eventos

astronómicos
"El sol tiene una forma de rotación parecida a la de la tierra"

"Otro tipo de evento es la rotación del sol, que se mueve mediante el paso del tiempo de las horas"
A partir de lo expuesto en la Tabla 2, se hace evidente que la forma en que los niños describen los eventos celestes se da por medio de interpretaciones o explicaciones halladas en los distintos medios de comunicación, éstos no dan una interpretación adecuada de la información que presentan, quizás debido al lenguaje que se maneja, pues los términos son técnicos y de difícil comprensión, generando así ideas que en la mayoría de casos no coinciden con lo que sucede en el mundo natural, promoviendo además nociones en los estudiantes que permanecen en el tiempo y que son difíciles de modificar (Cubero, 1989).

\section{Desde las preguntas}

Las inquietudes de los niños sobre el cosmos son diversas, por ejemplo, se interesaron por estudiar las galaxias, las estrellas, los planetas, la vida en otros planetas y la luna. Los ámbitos que mayor acogida tuvieron en la clase, fueron aquellos en los cuales no hay una explicación inmediata, como: ¿De dónde salió el agua del centro de la luna?; ¿por qué en algunos casos las estrellas cambian de color?, entre otros. Sus interrogantes llevaron a los estudiantes a buscar respuestas en fuentes como la televisión, la prensa, los libros, las películas, y lo que han aprendido en su escolaridad o escuchado de sus compañeros o adultos.

Se encontraron además inquietudes, como: ¿Qué hay en el lado oscuro de la luna?; ¿hay extraterrestres en el espacio? Este tipo de preguntas permiten pensar que su origen se encuentra en programas de investigación científica y películas de ciencia ficción; éstos han propiciado modos de explicar algunos eventos y han generado nuevas preguntas que no presentan una respuesta o explicación definida, tales como: ¿Los extraterrestres vendrán en son de paz o en son de maldad?; ¿por qué no hay gravedad en la luna?; ¿por qué el núcleo de la tierra es de fuego? Interrogantes que confirman el interés de los estudiantes por conocer más acerca de lo que sucede en el universo y que, en ocasiones, no son tratadas en la escuela, por lo que no tienen respuestas inmediatas y generan explicaciones nacidas de la experiencia y de fuentes como programas de investigación.

Los niños también tenían interés por indagar sobre la composición de algunos elementos que observan en el cielo o el espacio, como por ejemplo: ¿De qué están hechas las estrellas?; ¿de qué está hecha la luna?, preguntas que se repiten entre los grupos de estudiantes y que, en ocasiones, son contestadas desde la asimilación de lo que observan en la Tierra y de lo que escuchan de compañeros, como por ejemplo: "Hay astronautas en el espacio que viven en los satélites y los arreglan"; "En la parte clara de la luna hay huequitos", o "Esos huecos se llaman cráteres". Este tipo de interrogantes permiten generar un debate de la forma como se comprende el evento, al tiempo que enriquecer las explicaciones.

En la clase se pudo también dar cuenta de que las preguntas planteadas, individual y colectivamente, poseían todo tipo de forma: eran cerradas, abiertas e incitantes, como las plantean Giordan y De Vecchi (1995). En cuanto a las preguntas cerradas, en donde solo una respuesta es la adecuada, los niños se cuestionan por: ¿Cuál es el tamaño de la luna?; ¿el sol cuántos grados tiene?; ¿ ¿uándo se construyo el Curiosity?; ¿de qué está hecha la luna?, entre otras.

Las preguntas abiertas que son las que tratan un tema en general y permiten varias respuestas posibles, los niños se cuestionan asuntos, como: ¿Por qué se dice que Mercurio es el planeta más caliente y Plutón el 
más frío?; ¿Cómo se formó la luna antes de Cristo?; ¿Con qué se comunican los extraterrestres?; ¿cómo se crean los eclipses? En cuanto a las preguntas incitantes, que invitan a la búsqueda y a la profundización de un argumento, se distinguen algunas como: ¿Por qué en algunos casos las estrellas cambian de color?; ¿qué pasaría si un planeta se saliera de su órbita?; ¿por qué se movió la bandera de EEUU, cuando llegó el Apolo, si estaba en gravedad cero?, o ¿es posible que Plutón se pueda destruir por su núcleo frío?

\section{Desde las explicaciones T4}

Existen explicaciones de los estudiantes que se preocupan por dar respuesta a las causas que producen el evento astronómico, como la interacción de los cuerpos en el universo y la configuración de algunos eventos relevantes en el mundo natural en el que se encuentran inmersos y con el cual interactúan a diario. Un ejemplo de esto, se encuentra en la explicación de las causas que producen los movimientos de los planetas, es decir, la relación entre la fuerza electromagnética que producen los cuerpos, con la velocidad que poseen los planetas en su movimiento de translación.

Otros grupos, como el de vida extraterrestre, buscan explicar los eventos que implican la posible aparición de vida en otro cuerpo celeste diferente al planeta Tierra, encontrando que no existe evidencia de la existencia de ningún tipo de vida en otro lugar del espacio que no sea este planeta. También se presentan explicaciones relacionadas con el movimiento aparente de los astros observados desde la Tierra, en ellas se destaca que el motivo de la no visualización de las estrellas durante el día es la aparición del sol, y se puede intuir que para estos estudiantes las estrellas se encuentran siempre en la misma posición en el cielo, pasando por alto el movimiento de rotación de la Tierra. En la Tabla 3 se encuentran las explicaciones dadas por los estudiantes para explicar algunos fenómenos que les Ilaman la atención.

Tabla 3. Explicaciones generadas por los grupos de trabajo

\section{Grupo}

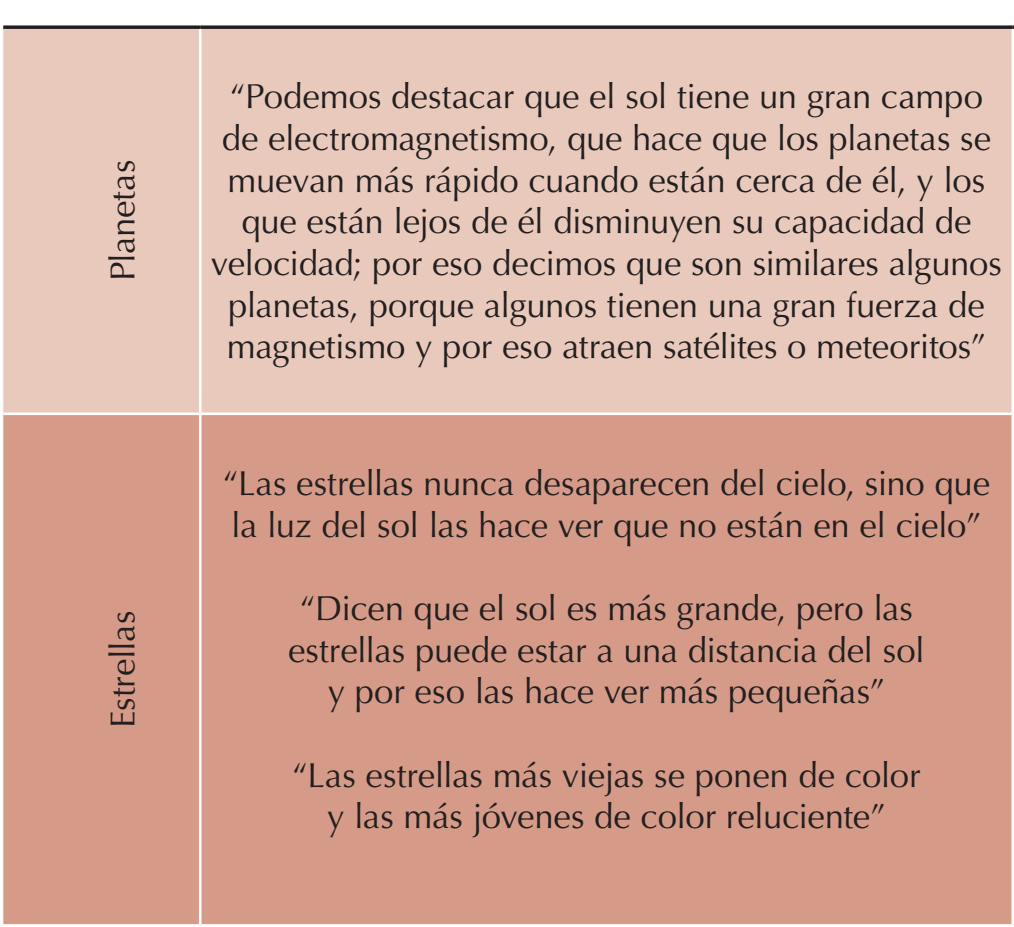

\section{Comentarios}

Se intuye una intención de observar la interacción de los planetas con los distintos cuerpos celestes que los rodean

Se nota una forma de exponer las causas por las cuales no se observan las estrellas en el día, y por qué se ven más pequeñas 


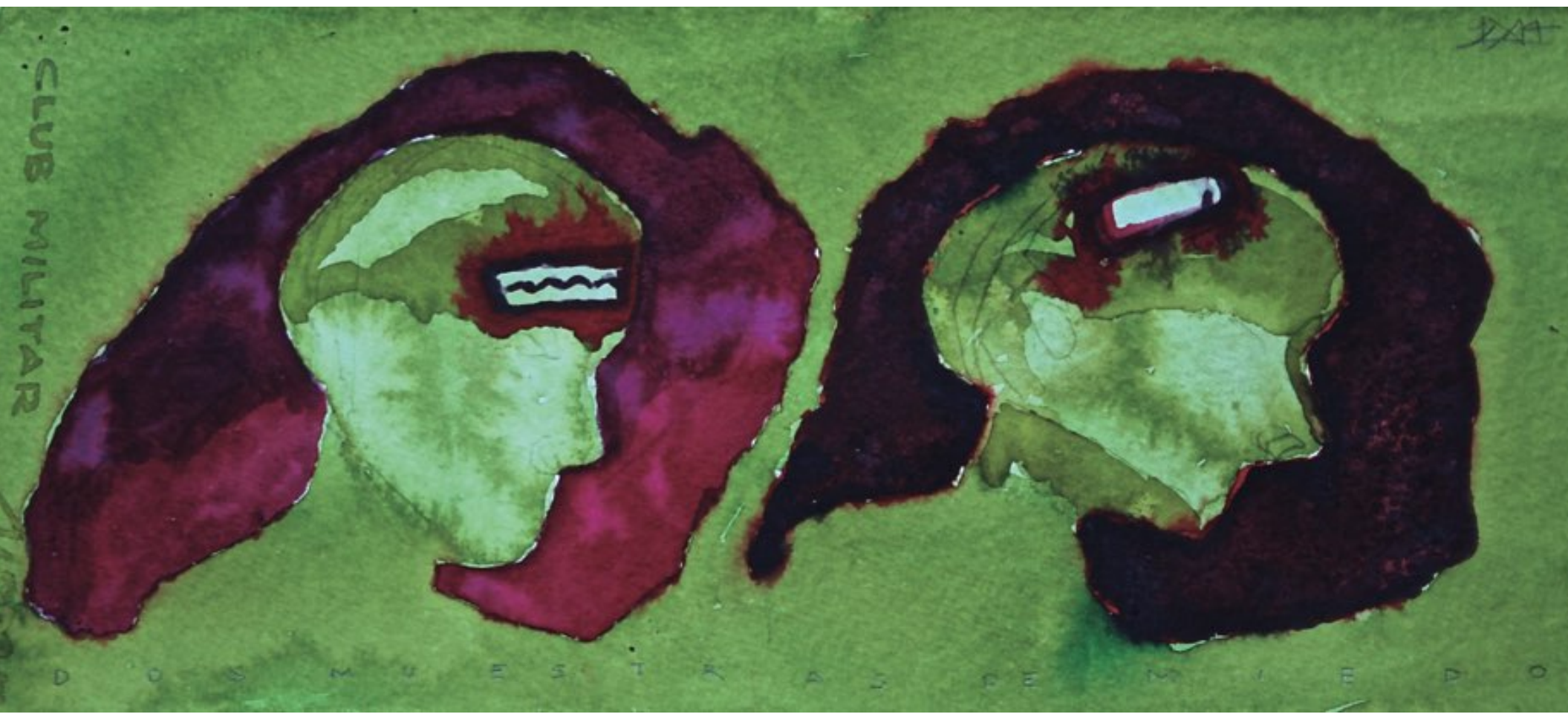

Luis Antonio Sánchez León ॥ Título: Dos Muestras de Miedo 》 Técnica: Acuarela ॥ Tamaño: 24.3 X 10.8 Cm 》 Año: 2014 


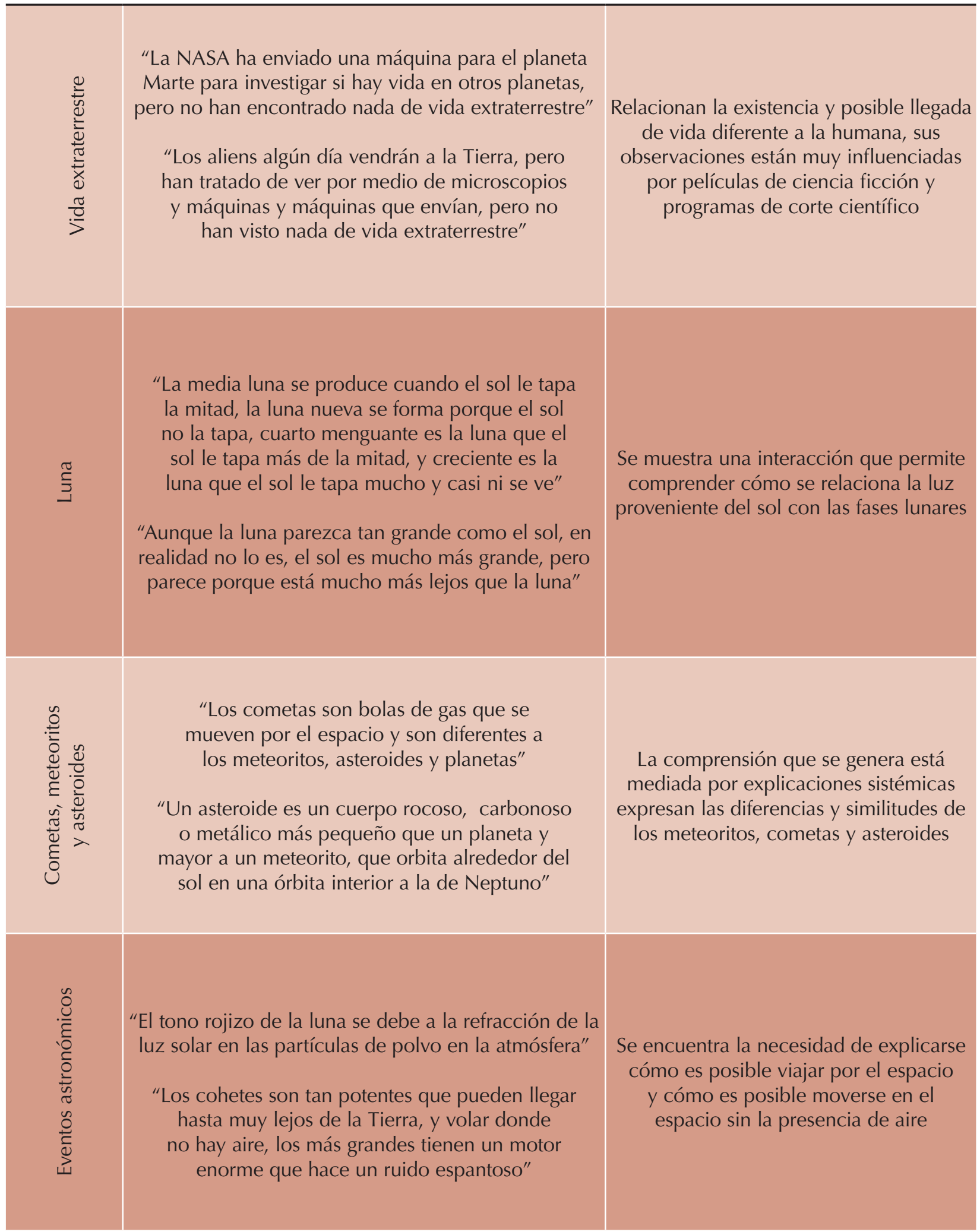




\section{Conclusiones}

Los eventos físicos y naturales, en particular los astronómicos, apasionan a los estudiantes, tienen una serie de experiencias que les permiten comprender lo que acontece en su entorno y de las cuales surgen nuevas inquietudes y explicaciones que, por lo general, no son tenidas en cuenta en la clase de ciencias. La vivencia en el aula hizo evidente que sin importar cuánto veamos el cielo de Bogotá, la curiosidad, el deseo de saber y la voluntad de conocer, nos hacen trascenderlo.

En su mayoría las inquietudes de los niños se orientaron hacia lo desconocido, lo mágico, el deseo de saber qué pasa más allá, en el firmamento, el mismo que está relacionado con su cotidianidad; también hay muchas preguntas que nacen de lo que ofrecen las películas, juegos o la propia clase de ciencias.

En la elaboración de explicaciones, se pudo distinguir un interés por temas como el movimiento de los planetas alrededor del sol, el aspecto de las estrellas (luminosidad, tamaño y tiempo de vida), la posibilidad de encontrar vida en un lugar distinto de la Tierra, y sobre la luna, particularmente sus cambios y regularidades de forma y tamaño.

Las explicaciones que emergieron son amplias y con distintos enfoques. Por ejemplo, cuando se preguntaron por el movimiento de los planetas alrededor del sol, los niños establecieron una analogía entre la fuerza de gravitación universal y la fuerza magnética que observan en los imanes. Consideraban que cuando los cuerpos estaban más cerca del sol se aceleraban, mientras disminuían su velocidad a medida de que se alejaban del mismo. La elaboración de historias, mitos y leyendas jugó un papel importante en la construcción de explicaciones; la tradición oral pasa de generación en generación y se transforma en explicaciones que tienen la misma validez que la ciencia para los niños.

Para generar la comprensión y el interés de los estudiantes, es necesario tener en cuenta sus inquietudes, ideas y explicaciones. Es importante para el maestro hacer una inmersión en el aula para enriquecer la mirada sobre la ciencia, en particular de la Astronomía y su enseñanza.

\section{Referencias}

Ayala, M. (2005). Análisis histórico-crítico y la recontextualización de saberes científicos. Construyendo un nuevo espacio de posibilidades. Pre impresos, 20, 1-17. Bogotá: Universidad Pedagógica Nacional.

Arias, G. G. (2006). La Astronomía y su enseñanza: posibilidades y perspectivas en el aula. Monografía de pregrado sin publicar. Bogotá: Universidad Pedagógica Nacional, Departamento de Física.

Barbosa, W., y Ramírez, A. (1995). La Astronomía y el movimiento. Monografía de pregrado sin publicar. Bogotá: Universidad Pedagógica Nacional, Departamento de Física.

Bautista, G., y Rodríguez, L. (1996). La ciencia como una actividad de construcción de explicaciones. Representaciones sobre la ciencia y el conocimiento, 2, 65-73.

Camino, N. (1999). Sobre la didáctica de la Astronomía y su inserción en la EGB.

Cubero, R. (1989). Cómo trabajar con las ideas de los alumnos. España: Diadas Editoras.

Giordan, A., y De Vencchi, G. (1995). Los orígenes del saber. Sevilla: Colecciones Investigación y Enseñanza Diada, Editoras S. L.

Molina, A. (2000). Conglomerado de relevancias de niños, niñas y jóvenes. Revista Científica, 4,1, 187-200.

Pérez Rodríguez, U. (2009). Utilización de los textos históricos y TICs en la enseñanza de la Astronomía: ¿la evidencia de los sentidos corrobora que la tierra es redonda? Alambique, 15(61), 57-64.

Rodríguez, G., Gómez, J., Flores, G., y Jiménez, E. (1996). Metodología de la investigación cualitativa. Málaga: Aljibe, D. L.

Ros, R. M. (2009). Abrir los ojos a la ciencia: Una razón para enseñar Astronomía. Alambique, 15(61), 9-17.

Sánchez, N. (1997). Una propuesta para la enseñanza de las ciencias a partir de actividades de Astronomía para niños. Monografía de pregrado sin publicar. Bogotá: Universidad Pedagógica Nacional, Departamento de Física.

Segura, D. (1993). La enseñanza de la física dificultades y perspectivas. Bogotá: Biblioteca de catedráticos.

Segura, D., Molina, A., y Pedreros, R. (1997). Actividades de investigación en la clase de ciencias. Sevilla: Diada Editora. rollos nacionales / Ideas, preguntas y explicaciones de $\mathbf{1 0 3}$ los niños sobre el cielo de Bogotá / Carlos Andrés Castañeda Sua / 


\section{Diálogo del conocimiento}

Con el artículo Ideas, preguntas y explicaciones de los niños sobre el cielo de Bogotá, el maestro Carlos Andrés Castañeda Sua nos invita a reflexionar sobre los modos como se aborda la ciencia en la construcción de conocimientos en el aula y si existe un diálogo de saberes encaminado a revitalizar la relación entre el niño y el mundo natural, dejándonos ver que con la enseñanza de la Astronomía nos acercamos a un cúmulo de posibilidades para fortalecer en los niños su "deseo de saber y voluntad de conocer", estimulando así la curiosidad como principio activo de la actitud investigativa.

En ese sentido, los tres referentes que nos plantea el autor: ideas, preguntas y explicaciones, conforman todo un tejido en función de recomponer la relación niño-conocimiento-mundo y constituye además una exigencia por reorientar las prácticas pedagógicas e investigativas de los docentes, estableciendo una ruptura frente a la tradicional educación bancaria que ya Freire puso en evidencia y cuestionó, donde los estudiantes son cuerpos vacíos que deben llenarse de contenidos, sin tener en cuenta su capacidad de interrogar e inventar el mundo. Frente a ello, el maestro Castañeda por medio de su investigación, nos muestra cómo a partir del encuentro con la Astronomía se pueden generar grandes oportunidades para que los niños participen en la construcción de conocimiento y se acerquen a la comprensión de la realidad.

De acuerdo con él, este ejercicio requiere que como maestros estemos dispuestos a conocer y dialogar con esa infinidad de ideas que traen los niños fruto de su proceso de socialización, activando así el reto de abrir un espacio significativo para la pregunta y la explicación, en razón a que los niños están inmersos en un flujo de información que arroja un sinnúmero de respuestas a preguntas que aún ellos mismos no se han formulado y que terminan asumiendo como verdades que configuran su saber. Al mismo tiempo nos lleva a cuestionar cómo se han validado los conocimientos que circulan en el aula y en la sociedad, que componen ese mar de verdades establecidas que poco espacio cede para la incertidumbre y la ensoñación, teniendo en cuenta que, como lo dice nuestro autor, para construir conocimiento la importancia radica no en la cantidad de información que se tiene sino en la explicación que se genera de esa información.

Al respecto, él también nos deja ver la influencia de los medios de comunicación en la configuración del saber, advirtiendo que si bien con ellos se moviliza en los niños ciertos intereses e inquietudes, estas al ser poco tratadas en la escuela quedan sin resolver, llevándoles de nuevo a buscar explicaciones en sus experiencias y en los mismos medios, los que en todo caso no ofrecen la claridad suficiente sobre la explicación de los sucesos, generando así una distorsión tanto en la idea como en la relación que se construye el niño con el mundo natural, efecto a remediar que constituye uno de los grandes retos de la enseñanza de las ciencias en la escuela.

De allí que, esta propuesta investigativa de Castañeda sobre el estudio de la astronomía con niños de quinto de primaria, surge como una posibilidad para recomponer esta alteración, en virtud de que con ella se abre paso a la magia de descubrir y soñar mundos posibles e inventar una mecánica del universo, lo que a la vez significa una reflexión sobre el mundo que tenemos y la comprensión de nuestro propio movimiento ligado al dinamismo de la Tierra. Igualmente, importa resaltar el carácter interdisciplinar que la enseñanza de la astronomía contiene, en razón de enriquecer al niño en su ser científico, tecnológico, artístico y socio afectivo, que además permite crear puentes entre el saber popular de raíces latinoamericanas y el saber académico propio de occidente, fortaleciendo la capacidad de dialogar con otras tradiciones para establecer relaciones complementarias en la construcción tanto de conocimiento como de identidad.

Yuletsy Gómez Garavito 